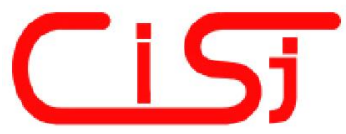

computing@computingonline.net www.computingonline.net
Print ISSN 1727-6209

On-line ISSN 2312-5381

International Journal of Bomputing

\title{
OBSERVATIONS-BASED COMPUTATIONAL ANALYTICS ON LOCAL CLIMATE DYNAMICS. PART 2: SEASONALITY
}

\author{
Yury Kolokolov, Anna Monovskaya \\ Orel State University, 95 Komsomolskaya str., 302026, Orel, Russia, www.oreluniver.ru \\ 2kolo@mail.ru, anna.monovskaya@mail.ru
}

\begin{abstract}
The paper continues the discussion concerning the computational decision making on evolution of local climate dynamics taking into account inevitable nonlinear nature of such systems and deficiency of reliable data on its dynamics. Here we focus on seasonality in the context of bifurcation phenomena described by the model of the hysteresis regulator with double synchronization (so-called HDS-model). From this conception, the method of structuring and analysis of meteorological data (method of relative scales) is proposed, where new useful information on local seasonal evolution becomes available. First of all, it concerns increase in analytical resolution (daily description in a climate scale). The key procedures of this method provide building the specialized seasonal structures in relative time scales. Advantages are illustrated in comparison with the traditional processing the time series of temperature observations on daily mean surface air temperature over last century. We believe that the results could be interesting in order to increase the confidence of estimations on coming climate changes. Copyright (C) Research Institute for Intelligent Computer Systems, 2017. All rights reserved.
\end{abstract}

Keywords: HDS-model, bifurcation analysis, climate data series, temperature observations, annual warming-cooling cycle, seasonal evolution.

\section{INTRODUCTION}

Per se, it seems to be impossible to imagine the contemporary climatology without IT-support in relation to collecting, digitizing, processing, transferring, storing, transmitting, converting and estimating climate data series [1]. Moreover, the point on enough IT-support becomes extremely pressing due to the combination between the inertia in official analytical methods and the observed increase in climate abnormalities [2, 3]. Why it occurs? Climate models relate, first of all, to middleterm (several decades at least) and long-term (paleoperiods of several centuries at least) time scales. In particular, the official standard climate norms remain calculated over 1961-1990 year [4, 5]. Regional and global weather models relate, first of all, to several days due to the "butterfly effect" $[6,7$, 8]. So, month and season estimations, especially with daily resolution and local peculiarities, remain comparatively far from the baselines of climate researches till now $[9,10,11,12,13,14]$. Theoretical fundamentals concerning seasonal evolution remain rather empirical due to ignoring the nonlinearities with evident hysteresis phenomena and feedbacks $[15,16,17]$. Let us briefly comment specific demands to methods of analysis in this case.
Simply, if a state of a nonlinear system is stable, then description of deviations from this state can be based on the statistical analysis. The current standard methods and protocols of meteorological data measurement and processing originate from this basis. The observed weather events exhibit growing deviations from the climate norms $[18,19,20]$. Similar facts mean that dynamics description should take into account bifurcation constituents [21, 22]. So, the standard data analytics have quite hard restrictions on its application [2, 15]. Taking into account both comparatively long duration and high monetary value which are necessary to spend for organization of new global climate observation network, it seems to be important to find novel ways on how to use the available data in order to get additional information. Then any new model and method of meteorological data analytics will need in IT-support in order to realize its verification at least; and such support supposes the specialized software, the main purpose of which is aimed at formalization of expert solutions.

From this viewpoint we focus hereafter on how to estimate local seasonal evolution. The novelty of the discussion is connected with the context of bifurcation phenomena described by the model of the hysteresis regulator with double synchronization 
(so-called HDS-model). This model provides unique chance to make daily descriptions of peculiarities of local annual temperature variation which are appropriated for heterogeneous local climate processes [23, 24, 25]. However it supposes reconsidering the traditional viewpoint [15]. With this purpose, we mention briefly the statistical seasonal estimations (section 2) and reveal contradictions for different time resolutions (section 3). Then we propose to avoid these contradictions at the expense of building the original seasonal structures in relative time scales. In section 4 we describe the idea of the corresponding analytics and illustrate typical examples. We use the open-access data of the meteorological observation on daily mean surface air temperature over last 100-130 years provided by Russian Research Institute of Hydrometeorological Information - World Data Center [26]. Main outcomes and future outlook are presented in section 5 .

\section{MODEL OF SEASONS IN THE ABSOLUTE SCALE}

Let us mention several important moments concerning notions of "seasonality", where climatology, ecology, geodynamics, and so on unite to classify regional changes. In other words, some seasonal patterns are observed in local dynamics, where each of them is visualized by the corresponding changes in flora and fauna [23, 27, 28]. The climatology viewpoint $[19,27,28]$ describes these circumstances by four seasons, which lasts three months in the context of so-called annual temperature variation (hereafter ATV-unit) from January to January by monthly means (TмоNтH). In the case of the Northern hemisphere, the following division occurs (Fig.1a): "spring" is from March to May; "summer" is from June to August; "autumn" is from September to November; "winter" is from December to February. Peculiarities of seasonal patterns relate to regions.

Then two assumptions are supposed. First, seasons are accepted as regular ranges in time within a year; climate states and norms are estimated over regular time windows. Hereafter we base on the current meteorological convention, in accordance to which time windows follow in 30-years with fixed beginning dates (Fig.1b, regular widows). Second, deviations from the norms are excused by chaotic constituent and are statistically described. So, in general, formal traditional seasonal estimations are made in accordance with the following main procedures (Fig.1c): specialized data structuring; calculation of climatic states and norms; estimating seasonal dynamics or/and abnormalities. Due to the best combination of both duration and correctness of the instrumental measurements, temperature observations remain the main variable for analytics $[5,29,30]$, and we restrict our discussion by the land surface air temperature.

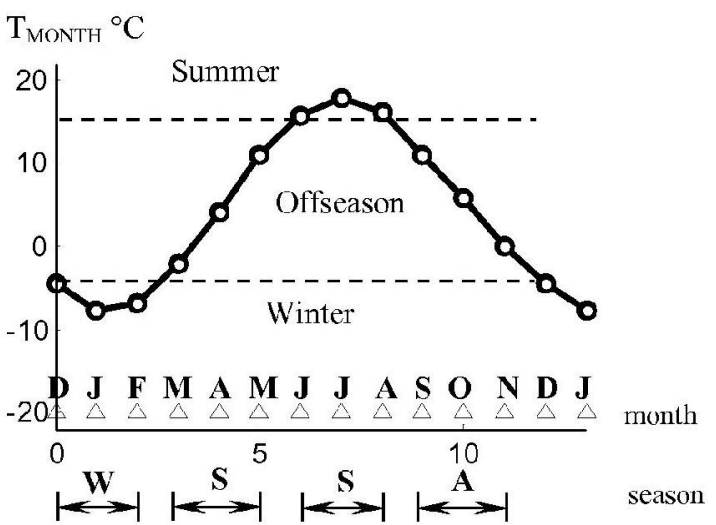

(a)
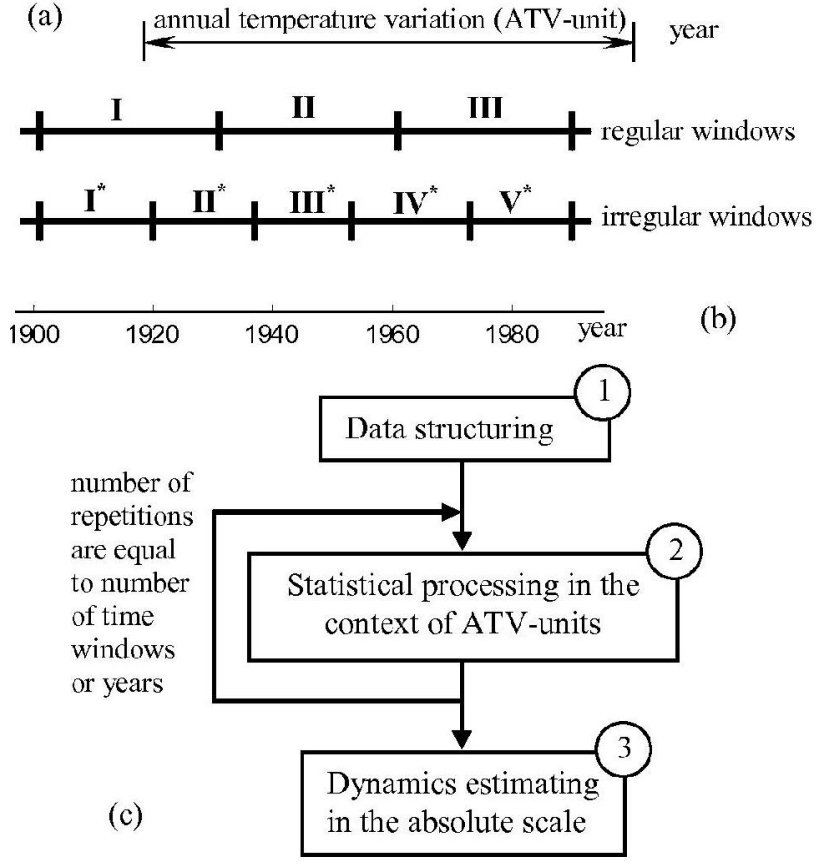

Fig. 1 - To notions of "seasonality" (a); comparative division on regular and irregular time windows (b); the main cycle of data analysis in the absolute scale (c). Examples here and after are illustrated for St.Petersburg (WMO-code 26063).

Official protocols of data observation, measuring, translation and filing represent the basis of the first procedure. In particular, the Global Climate Observation Network remains the main provider of the unified hydro-meteorological observations, which are collected and processed within the specialized departments of the State Federal Services for Hydrometeorology and Environmental Monitoring. With research purposes, data from the official websites are translated to forms which are proper for original software. In particular, we translated data got from [26] into specialized 3Dstructures for each local climate system. Such structure for St.-Petersburg in (day, $T_{D A Y}$, year)- 
coordinates is visualized in Fig.2a, where $\mathrm{T}_{\mathrm{DAY}}$ is a daily mean temperature.
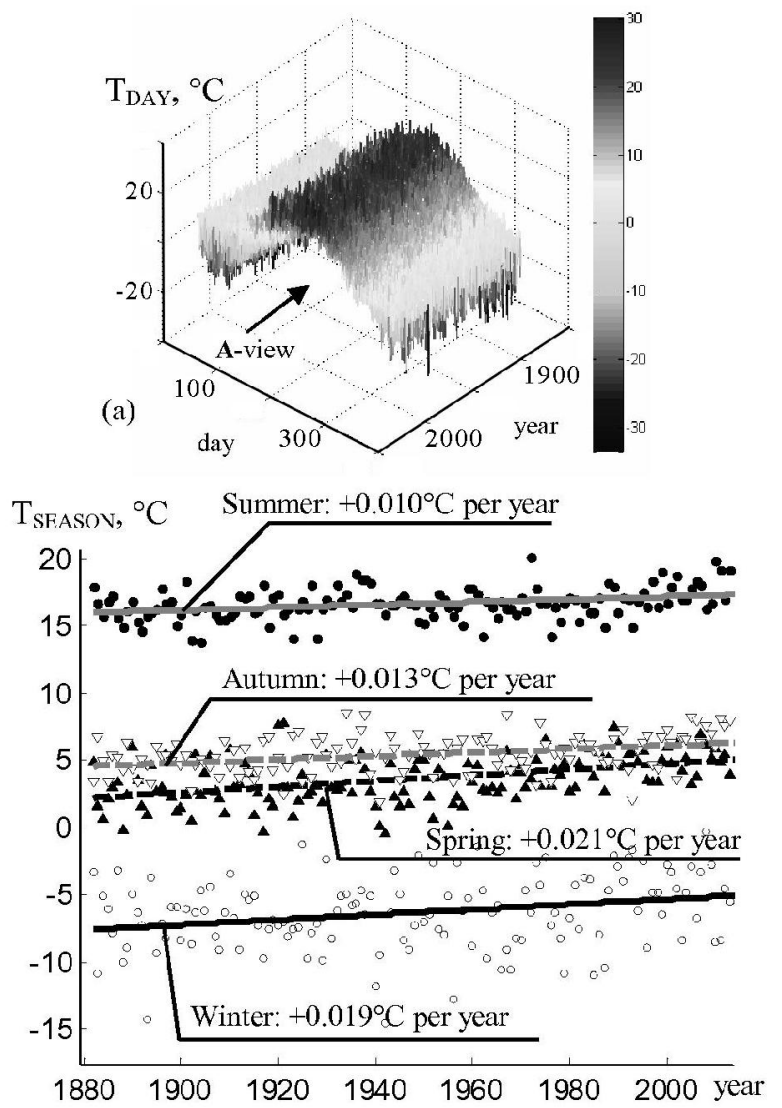

(b)
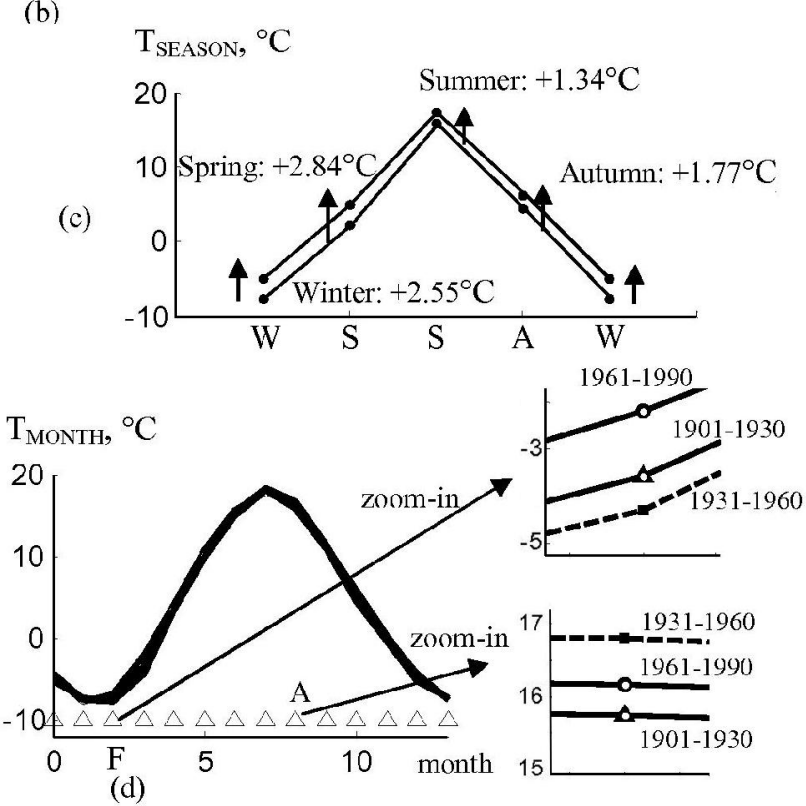

Fig. 2 - 3D-structure of the meteorological data (a); seasonal dynamics over 1881-2015 years presented by time series with trends (b) and by the linear seasonal pattern (c); monthly dynamics of annual temperature variation over 1901-1990 years (d).

The second procedure is based on statistical processing the local observations in the context of ATV-units. The norms are traditionally calculated for the reference time window of 1961-1990 years. Last years, other durations and other beginning dates are also considered, for example [5, 20, 24]. However, all these variants continue to take into account the mentioned assumption on common regular time windows for all the local climate systems. So, a normal climate dynamic at a certain place over a quasi-homogeneous climate region is presented in the average form for each regular window (similar to the pattern presented in Fig.1a), and it is the third assumption that each local climate norm follows by one kind of annual behavior [4, 20].

Trend analysis of the data as well as estimating extremes and deviations from norms are realized during the third procedure. These calculations relate mainly to monthly and seasonal resolutions, where seasonal trends demonstrate quite good correlation with the trends of regional and global warming [5, 18, 19]. For example, seasonal trends are illustrated in Fig.2b. Here the increase in wintry, spring, summer and autumnal average temperatures occurs, where the corresponding total temperature changes per season are summarized in Fig.2c. Once further specification occurs, tendencies are not so clear [3, 18]. For example, let us compare monthly ATVunits over three successive time windows (Fig.2d): 1901-1930 years; 1931-1960 years; 1961-1990 years. Then different tendencies can be observed. For example, February (F) is the coldest and August (A) is the warmest over 1931-1960 years (zoom-in fragments in Fig.2d).

And else one moment seems to be important limits on duration of the reliable observations. For example, only three whole 30-year cycles occur over a century (Fig.1b), where it is impossible to build standard climate norms as before 1900 year (due to data before 1881 are absent, and these data are already irreplaceable) as after 1990 year (due to it is necessary to wait the corresponding results of measurements during several years). That is why search of ways to get more useful information from the available data are pressing.

\section{RELATIVE SEASONALITY}

So, the traditional conception of regular windows is based on the assumptions and restrictions which become to be reconsidered and discussed [2, 3, 4, 25]. For example, let us calculate the following (Fig.3a): mean daily values (white central line) and deviations (gray up and low lines to show $\pm 3 \sigma$ deviations from the means, where $\sigma$ is a standard deviation). Then a lot of statistical misses occur (black points beyond gray limits in zoom-in fragments to Fig.3a). It means that there is at least one qualitative change of local climate behavior over the considered period. Let us stretch the structure with daily resolution along time axis (Fig.3b) and estimate trends of annual maximums and minimums 
(gray circles in Fig.3b and Fig.3c). Then the magnitudes of total temperature changes (Fig.3d) reconstructed in accordance with the trends (Fig.3c) are essentially different from the ones built on the basis of the seasonal trends over the same time (Fig.2c). The difference in wintry estimations is about $20 \%$ and the difference in summer estimations is about $70 \%$; the difference in the annual amplitudes is about $50 \%$. It initiates reasoning concerning alternative models and methods of the meteorological data analytics.

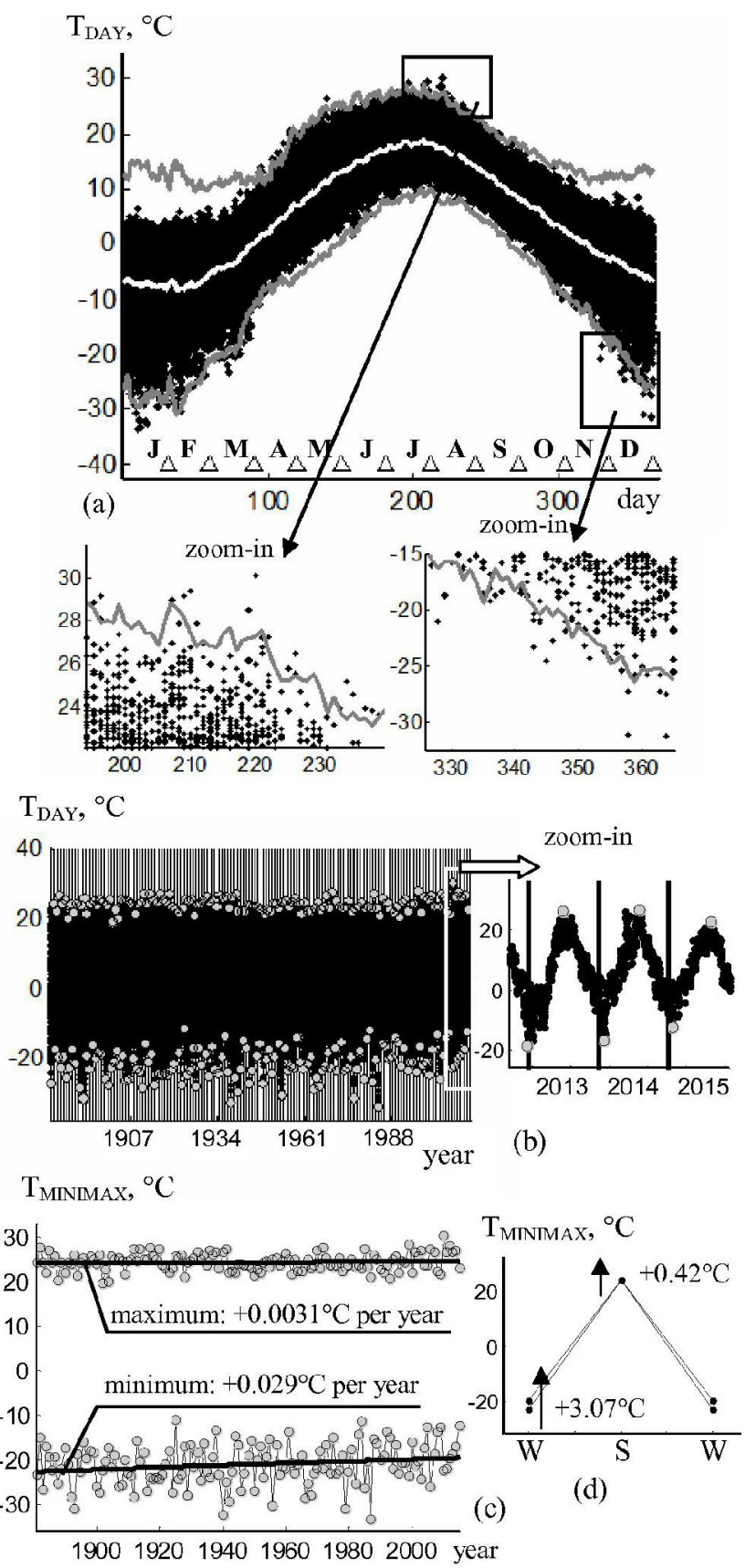

Fig. 3 - Processing the temperature observations over 1881-2015 years: result of the statistical presentation of 3D-structure (Fig.2a) with zoom-in fragments of misses (a); time series with minimax annual points (b) with its trends (c) and linear structure of the total shifts (d).
We paid attention on this moment from the practical considerations. If averaged per month (Fig.1a) or per day (Fig.3a) values are used, then ranges of wintry and summer temperatures are smaller than off-seasonal ranges. So, if essential increase in wintry temperature is observed, then the corresponding increment $\left(+3.07^{\circ} \mathrm{C}\right.$, Fig.3d) can be comparable with the wintry range (Fig. 1a). In other words, the habitual "winter"-range can disappear, and rather bi-seasonality appears with another "summer" and another "off-season". However, what does it mean "another"? Answer on this question is absent with the traditional viewpoint on one pattern of annual behavior with fixed seasonal ranges (Fig.1a). That is why we develop the tools (models, methods and software) for climate dynamics analytics in the context of bifurcation phenomena described by the model of the hysteresis regulator with double synchronization (HDS-model). Mathematical description of HDS-model and its verification by data of temperature observations were presented in $[15,25]$. Let us comment briefly peculiarities of HDS-model dynamics which are significant for the discussion.

The HDS-model is characterized by a variable structure, where the moments of structural changes are determined by four surfaces (Fig.4a): two surfaces (denoted by " 1 " and " 3 ") sew the phase trajectories by level; two surfaces (denoted by "2" and " 4 ") sew the phase trajectories by time. The time quantization is realized by $\mathrm{F}_{1}$ - and $\mathrm{F}_{2}$-sequences with equal frequency $\left(1 / \mathrm{T}_{\mathrm{S}}\right)$ and $\mathrm{T}_{\mathrm{S}} / 2$-shift in time, where $T_{S}$ corresponds to one year. Then, the baseline dynamics of a local climate system represents alternations between three elemental processes with the same periodicity $\left(T_{S}\right)$ and different orders of its structural changes (Fig.4a): "right"-process by 3-24-3 sequence with $k>0.5$ ( $R$-behavior); "central"process by $2-4-2$ - sequence with $k \approx 0.5$ ( $C$-behavior); "left"-process by 2-1-4-2 sequence with $k<0.5$ ( $L$ behavior). Here $k$ is a relative duration of a warming stage within each annual warming-cooling cycle (AWCC-unit [15]).

So, in accordance with HDS-model, a local climate dynamics should be described by a multibehavior model, where three kinds of annual behavior should be collected (so-called, RLCensemble (Fig.4b) in contrast to the traditional unibehavior model (Fig.1a). Let us use the multibehavior model for a novel method to estimate seasonal evolution.

\section{METHOD OF RELATIVE SCALES}

While description of seasonality in accordance with HDS-model, it becomes necessary to take into account relations between several scales: $R-, L-, C$ - 
behaviors are caught by sewing surfaces (dash-dot lines in Fig.4b); AWCC-unit relates to the temperature minimum (black two-side arrow in Fig.4c); seasons relate to regular division in three months (Fig.4c, gray two-side arrows). Here the dimensional correlations used by schemes are based on generalization of the results of reconstruction of $R L C$-ensembles made for 39 local climate systems $[3,18]$. Then $R-, L-, C$-behaviors form a seasonal domain (filled by gray in Fig.4c), where there are two wintry peaks and two summer peaks (denoted by ovals). Intersections between seasonal limits (gray vertical lines) and limits of a seasonal domain show seasonal temperature ranges (denoted by gray, white and black colored rectangles on the right of Fig.4c). Also, one moment should be taken into account, namely: $R L C$-ensemble can consist of stable and unstable behaviors. This situation likes multiple attractors which are typical for systems with a variable structure [21, 25]. Usually $L$ behavior or $R$-behavior can be unrealized, and it means that the corresponding phase structure is rather unstable (denoted by dotted lines in Fig.4d,e correspondingly). However, such structure can initiate rare abnormal deviations from the observed annual norms.

So, the idea of the method consists in the following: seasonal temperature ranges of different local climate systems can be compared with daily resolution. Then beginning dates of the same seasons are usually different in more or less extent. Taking into account that HDS-model operates with particular irregular time windows for each local climate system $[15,25]$, reconstruction of seasonal dynamics becomes nontrivial task. Nevertheless, such analytics could be formalized in general [3, 18]. Of course, the algorithms of meteorological data processing become more complex in comparison with the ones traditionally used. For example, let compare the proposed main cycle (Fig.5) with the traditional one (Fig.1c). Per se, it is a point of two additional procedures (the specialized bifurcation analysis and ensemble clustering), extensions for existing statistical processing and formalization of the described idea of expert seasonal estimations.

The specialized bifurcation analysis is made in the context of the peculiarities of HDS-model dynamics and its procedures are detailed in [25]. As a result, evolution of local climate systems becomes reasonably divided by irregular windows. In other words, a uniqueness of changes in dynamics of each local climate system is taken into account $[18,25]$. For example, over the considered period, there are three regular windows and five irregular windows (Fig.1b). The ensemble clustering is used in order to build a unique $R L C$-ensemble for each time window. In particular, we use the clustering in the context of
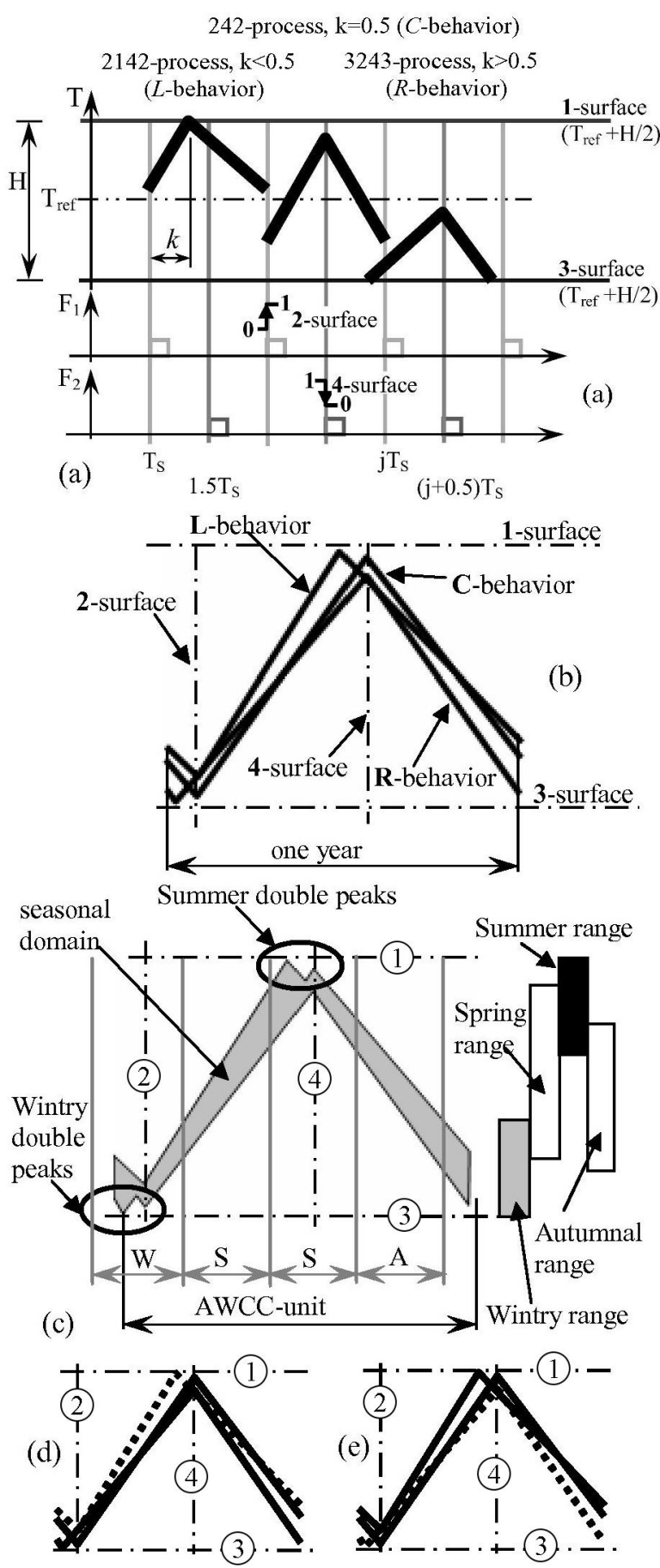

(1) 1-surface (2) 2-surface (3) 3-surface (4) 4-surface

Fig. 4 - To peculiarities of HDS-dynamics (a); schemes to explain the ensemble estimating of seasonality: $R L C$-ensemble (b); seasonal pattern in relative scales (c); $L$-instability (d) and $R$-instability (e) in comparison with the general view (b).

peculiarities of the linearized annual dynamics [18]. Since each ensemble is built for each time window, then the statistical processing repeats three times (the internal procedure in Fig.5). Last, the cycle includes the estimating in relative scales (Fig.4c) taking into account stable and unstable constituents of each $R L C$-ensemble (Figs.4d,e). So, we realize the procedures Fig.5 on MatLAB platform and fulfill 
test calculations in order to get first seasonal estimations from HDS-model.

Let us comment typical results of the test estimations (Fig.6) in comparison with the traditional viewpoint (Fig.2b,c,d). Let the first and last irregular windows (Fig.1b) be considered. Let the absolute scale be denoted by day-axis and relative seasons be denoted by abbreviation of its first letters in Fig.6. In the first case (Fig.6a) summer double peaks are practically reduced to one; in the second case the both peaks are distinguished quite clear (Fig.6b). Increase in temperature maximum is about $0.5 \ldots 1^{\circ} \mathrm{C}$ (Fig.6c) that is concordant with the observations (Fig.3d). Next, in the first case there are clear wintry double peaks (Fig.6a); in the second case such peaks reduced to one, which is shifted towards the end of January (Fig.6b). Increase in temperature minimum is about $3{ }^{\circ} \mathrm{C}$ (Fig.6c) that is concordant with the observations (Fig.3d). The dotted lines in Fig.6b denote the unstable limit cycle of $L$-behavior, to which the current annual warmingcooling cycle can abruptly deviate during more or less time. Such double peaks and unstable abnormalities can not be originally estimated from the traditional viewpoint.

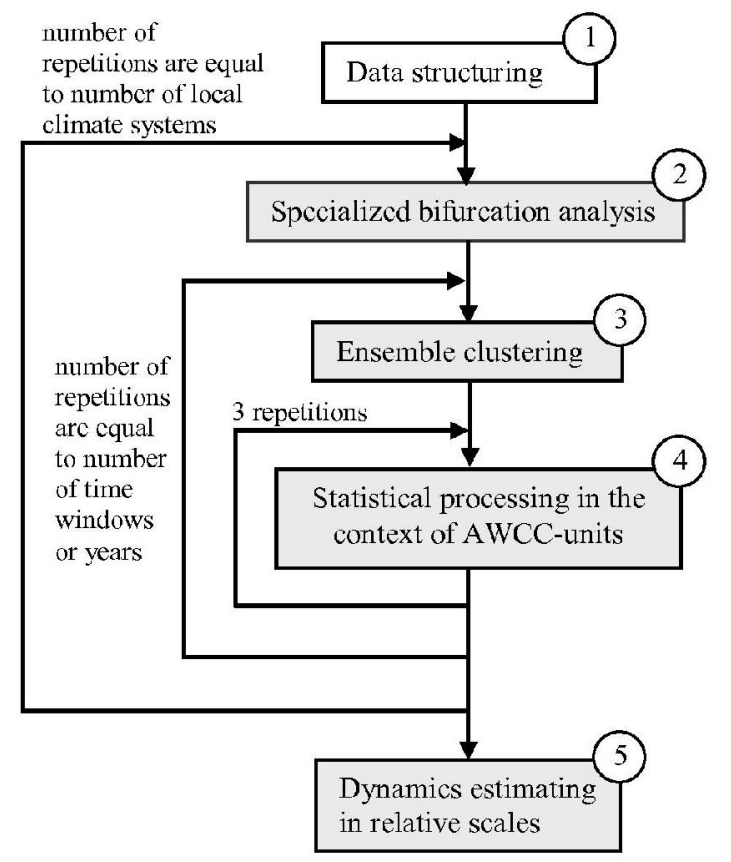

Fig. 5 - The main cycle of data analysis in relative scales.

At the same time, evolution of seasonal ranges can be visible by comparison of these cases (Fig.6c). First, the essential off-seasonal asymmetry is observed at present in comparison with the past. Next, abrupt falls of temperature can occur during Autumn and Winter (denoted by stripy domains). So, the difference between the astronomic year (the absolute scale) and each local "seasonal" year (the relative scale) can be visualized and estimated within the testing viewpoint and is latent for the traditional viewpoint (Fig.2b,c,d).

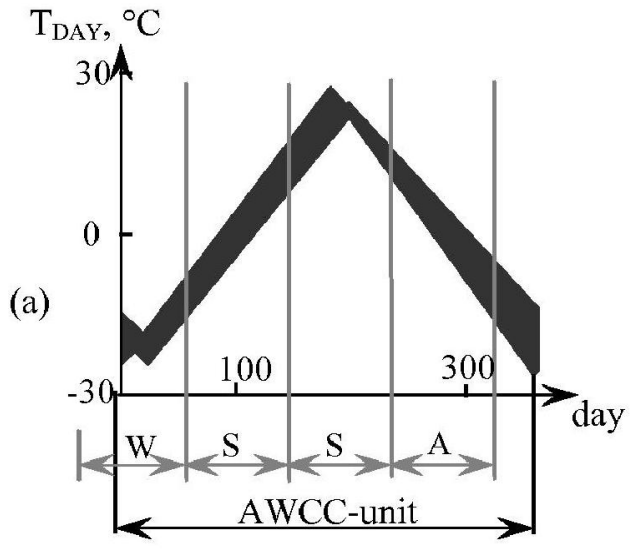

$\mathrm{T}_{\mathrm{DAY}},{ }^{\circ} \mathrm{C}$

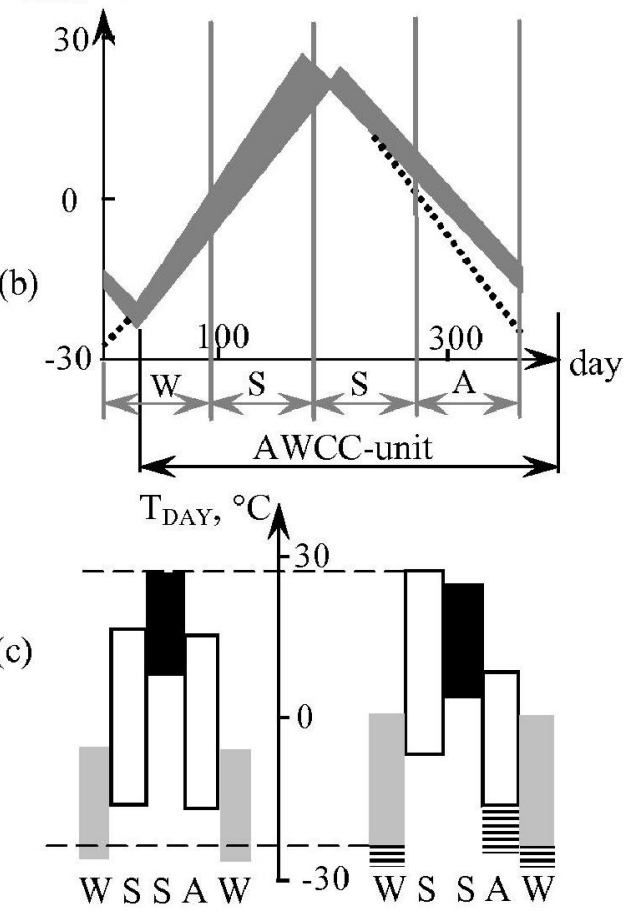

Fig. 6 -Patters of relative seasons built for the begin (a) and for the end (b) of the 20 century with comparative estimation of its structures (c).

\section{CONCLUSION}

Per se, the paper discusses the new method of time series analysis which is oriented on specialized structuring and analysis of daily mean temperature observations (not simulations) in climate scale (during several decades and more). The specialization is theoretically grounded on the peculiarities of nonlinear dynamics of the HDSmodel which is recently used in order to describe conceptually local climate dynamics in various practice-oriented applications $[3,15,18]$. Concerning the discussed issue, the main advantage of the HDS-model is connected with the ability to increase analytical resolution. Here we presented 
first test results on how to analyse seasonality in such context. With this purpose we propose and formalize the idea of relative scales, realize the necessary procedures on MatLAB platform and fulfill test calculations. The results demonstrate new useful information on qualitative and quantitative characteristics of seasonal evolution in comparison with the monthly mean seasonal model. Since HDSmodel is physically grounded and verified $[15,18$, 25], then we believe that the proposed method could be used in order to increase the confidence of estimations on coming climate change including generating unconventional abnormal scenaria which are theoretically possible. The main restriction on practical applications is connected with temperature analytics only.

\section{REFERENCES}

[1] S. Ribeiro, J. Caineta and A. C. Costa, "Review and discussion of homogenization methods for climate data," Physics and Chemistry of the Earth, vol. 94, pp. 167-179, 2016.

[2] Ch. Essex, "Climate theory versus a theory for climate," International Journal of Bifurcation and Chaos, vol. 21, pp. 3477-3487, 2011.

[3] Yu. Kolokolov and A. Monovskaya, "Guesswork and reasonings on centennial evolution of surface air temperature in Russia. Part IV: Towards economic estimations of climaterelated damages from the bifurcation analysis viewpoint?" International Journal of Bifurcation and Chaos, vol. 26, 1630033, 2016.

[4] A. Argues and R. S. Vose, "The definition of the standard WMO climate normal: The key to deriving alternative climate normals," Bulletin of American Meteorological Society, vol. 92, pp. 699-704, 2011.

[5] T. F. Stoker, et al., "Summary for policymakers," in: Climate Change 2013: The Physical Science Basis. Contribution of Working Group I to the Fifth Assessment Report of the Intergovernmental Panel on Climate Change [Stocker, T.F., D. Qin, G.-K. Plattner, M. Tignor, S.K. Allen, J. Boschung, A. Nauels, Y. Xia, V. Bex and P.M. Midgley (eds.)]. Cambridge Univ. Press, Cambridge, UK and NY, USA, 2013.

[6] D. R. Novak, C. Bailey, K. F. Brill, P. Burke, W. A. Hogsett, R. Rausch and M. Schichtel, "Precipitation and temperature forecast performance at the weather prediction center," Weather and Forecasting, vol. 29, pp. 489-504, 2014.

[7] A. Trevisan and L. Palatella, "Chaos and weather forecasting: The role of the unstable subspace in predictability and state estimation problems," International Journal of Bifurcation and Chaos, vol. 21, pp. 3389-3415, 2011.

[8] F. Uboldi and A. Trevisan, "Multi-scale error growth in a convection-resolving model," Nonlinear Processes in Geophysics, vol. 22, pp. 1-13, 2015.

[9] J. A. Dutton, R. P. James and J. D. Ross, "Calibration and combination of dynamical seasonal forecasts to enhance the value of predicted probabilities for managing risk," Climate Dynamics, vol. 40, pp. 3089-3105, 2013.

[10] http://effis.jrc.ec.europa.eu/applications/season al-forecast/

[11] www.cpc.ncep.noaa.gov/products/predictions/ long_range/two_class.php

[12] http://weather.gc.ca/saisons/prob e.html

[13] www.hko.gov.hk/wxinfo/season/season.htm

[14] http://wmc.meteoinfo.ru/season

[15] Yu. Kolokolov and A. Monovskaya, "Guesswork and reasonings on centennial evolution of surface air temperature in Russia: Is it possible to build bifurcation diagrams based on extrashort local observations?," International Journal of Bifurcation and Chaos, vol. 26, 1650071, 2016.

[16] J. M. Thompson and J. Sieber, "Predicting climate tipping as a noisy bifurcation: A review," International Journal of Bifurcation and Chaos, vol. 21, pp. 399-423, 2011.

[17] R. A. Colman, "Climate radiative feedbacks and adjustments at the Earth's surface," Journal of Geophysics Research: Atmosphere, vol. 120, pp. 3173-3182, 2015.

[18] Yu. Kolokolov and A. Monovskaya, "Guesswork and reasonings on centennial evolution of surface air temperature in Russia. Part III: Where is the joint between norms and hazards from the bifurcation analysis viewpoint?," International Journal of Bifurcation and Chaos, vol. 26, issue 6, 1650122, 2016.

[19] J. Blunden and D. S. Arndt (Eds.), State of the Climate in 2015, Bulletin of American Meteorological Society, vol. 97, issue 8, S1S275, 2016.

[20] Documents of the 16th Session of the Commission for Climatology (CCl-16), Heidelberg, Germany, July 3-8, 2014: CCL16/Doc.8.1. Final Report with resolutions and recommendations (WMO-No.1137, World Meteorological Organization, Geneva, Switzerland).

[21] Yu. Kolokolov and A. Monovskaya, "From modifications of experimental bifurcation diagrams to operating process stability margin," International Journal of Bifurcation and Chaos, vol. 23, 1330024, 2013. 
[22] Yu. Kolokolov and A. Monovskaya, "Modified bifurcation diagrams to analyse the intermittency observed in local climate dynamics," in Proceedings of the $8^{\text {th }}$ IEEE International Conference on Intelligent Data Acquisition and Advanced Computing Systems: Technology and Applications (IDAACS'2015), Warsaw, Poland, September 24-26, 2015, pp. 624-630.

[23] N. B. Grimm, F. S. Chapin III, B. Bierwagen, P. Gonzalez, P. M. Groffman, Y. Luo, F. Melton, K. Nadelhoffer, A. Pairis, P. A. Raymond, L. Schimeland, and C. E. Williamson, "The impacts of climate change on ecosystem structure and function," Frontiers in Ecology and the Environment, vol. 11, pp. 474-482, 2013.

[24] G. J. Boer, V. V. Kharin and W. J. Merryfield, "Decadal predictability and forecast skill," Climate Dynamics, vol. 41, pp. 1817-1833, 2013.

[25] Yu. Kolokolov and A. Monovskaya, "Guesswork and reasonings on centennial evolution of surface air temperature in Russia. Part II: Is it possible to research both local peculiarities and regional tendencies from the bifurcation analysis viewpoint?," International Journal of Bifurcation and Chaos, vol. 26, 1650071, 2016.

[26] www.meteo.ru/english/climate/cl_data.php

[27] A. K. Betts, "Seasonal climate transitions in New England," Royal Meteorological Society: Weather, vol. 66, no. 9, pp. 245-248, 2011.

[28] N. Torbick, B. Ziniti, S. Wu and E. Linder, "Spatiotemporal lake skin summer temperature trends in the Northeast United States," Earth Interactions, vol. 20, pp. 20-25, 2016.

[29] T. R. Knutson, F. Zeng and A. T. Wittenberg, "Multimodel assessment of regional surface temperature trends: CMIP3 and CMIP5 twentieth-century simulations," Journal of Climate, vol. 26, pp. 8709-8743, 2013.

[30] J. Feng, $\mathrm{Z}$. $\mathrm{Wu}$ and G. Liu, "Fast multidimensional ensemble empirical mode decomposition using a data compression technique," Journal of Climate, vol. 27, pp. 3492-3504, 2014.

[31] M. B. Soares and S. Dessai, "Exploring the use of seasonal climate forecasts in Europe through expert elicitation," Climate Risk Assessment, vol. 10, pp. 8-16, 2015.

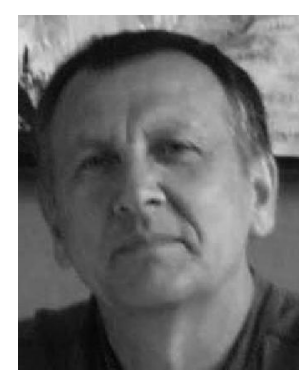

Yury Kolokolov received M.S. degree in 1972 (Tomsk Politechnical Institute, Russia), PhD degree in 1978 (Tomsk Politechnical Institute, Russia), Russian academic degree of Senior Doctor in 1990 (Tomsk University of Control and Radioelectronics, Russia).

From 1993 to 2007 he was a full professor and Head of the Dept. of Design and technology of electronic systems (State Technical University of Orel, Russia); from 2007 to 2012 he was a full professor and Director of Institute of Control Systems and Information Technologies (UGRA State University, Khanty-Mansiysk, Russia); from 2012 to 2016 he was a chief researcher and a Head of Dept. of Applied Scientific Research (Russian Research Institute of Hydro meteorological Information - World Data Center, Obinsk, Russia). Currently he is a Head of scientific school (Orel State University, Russia). His scientific interests include theory, experiment and practice of nonlinear dynamics in the field of complex systems.

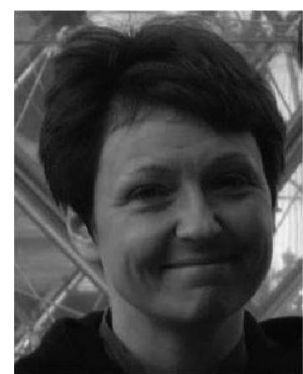

Anna Monovskaya received M.S. degree in 2001 (State Technical University of Orel, Russia), PhD degree in 2005 (State Technical University of Orel, Russia and University of Technology of Troyes, France), Russian academic degree of Senior Doctor in 2010.

Currently, she is a Head of a ScientificEducational Center (Orel State University, Russia). Her scientific interests include theory, experiment and practice of nonlinear dynamics in the field of complex systems. 